Revista Científica de FAREM-Estelí

Medio ambiente, tecnologia y desarrollo humano

Año 10 | Edición especial: artículos de revisión documental

ISSN: 2305-5790

https://rcientificaesteli.unan.edu.ni

DOI: https://doi.org/10.5377/farem.v0i0.11608

\section{Importancia del aprendizaje significativo en la construcción de conocimientos}

\section{Importance of significative learning in the construction of the knowledge}

\section{Julio César Roa Rocha}

UNAN-Managua, FAREM-Matagalpa

https://orcid.org/0000-0002-4505-7698

juliorn27@yahoo.com
RECIBIDO

$26 / 03 / 2021$

ACEPTADO

19/052021

\section{RESUMEN}

El siguiente artículo presenta una revisión bibliográfica sobre el aprendizaje significativo, propuesto por David Ausubel, en la que se describe su significado, naturaleza, y características, con el objetivo de analizar su valor en la educación superior. Igualmente, se explican las características de un material de aprendizaje potencialmente significativo, tipos de aprendizajes significativos y la adquisición del conocimiento. Se parte de la premisa de que el aprendizaje significativo surge cuando el alumno, como constructor de su propio conocimiento, relaciona los conceptos a aprender y les da un sentido a partir de la estructura conceptual que ya posee. De este modo, construye nuevos conocimientos al relacionar los conceptos nuevos con la experiencia que ya tiene. Este artículo aborda de manera particular la pertinencia de este tipo de aprendizaje aplicado a la educación superior, específicamente a la UNAN MANAGUA. Con tal fin, se aplicó la investigación documental, como una técnica de investigación cualitativa, exploratoria y analítica para la recolección de información relevante sobre la importancia del aprendizaje significativo en la construcción de conocimientos, de este modo se recopiló y seleccionó información mediante la lectura de documentos, libros, revistas, periódicos, bibliografías, etc. Se llegó a la conclusión de que el aprendizaje significativo es un reto y una oportunidad para la construcción de los nuevos saberes, lo que implica la necesidad de cambios estructurales en la educación superior para cualificar a los futuros profesionales con actitud crítica, propositiva y reflexiva. 


\section{ABSTRACT}

This following article presents a bibliographic review on significative learning proposed by David Ausubel, describing it's meaning, nature, and characteristic, with the objective of analyzing its value in higher education. Likewise, the characteristics of potentially significant learning material, types of significative learning, and the acquisition of knowledge are explained. It is based on the premise that the significative learning arises when the student, as the constructor of self-knowledge, relates the concepts to be learned and gives them a meaning based on the conceptual structure the student already possesses. In this way, the student construct new knowledge by relating new concepts to the experience they already have. This article approaches in a particular way the relevance of this type of learning applied to higher education, specifically to UNAN-Managua. To this end, documentary research was applied, as a qualitative, exploratory, and analytical research technique for the collection of relevant information on the importance of significative learning in the construction of knowledge, thus, information was collected, and selected by reading documents, books, journal, newspaper, bibliographies, etc. It was concluded that significative learning is a challenge and an opportunity for the construction of the new knowledge, which implies the need for structural changes in higher education in order to qualify future professionals with a critical, proactive and reflective attitude

\section{KEYWORDS}

Knowledge Acquisition; significative learning; constructivism; approach; pedagogy; bibliographic review. 


\section{INTRODUCCIÓN}

La educación en general, y en particular la educación superior, ha enfrentado diferentes cambios y desafíos a lo largo de su historia, como producto de las necesidades y demandas sociales, que incitan de alguna manera a la adaptación a las nuevas realidades del siglo XXI.

Hasta hace unos años, en el contexto de la educación nicaragüense, era común observar que los estudiantes aprendieran grandes volúmenes de información de manera memorística. Su trabajo consistía en almacenar información abundante, a través de un esfuerzo complejo y de concentración mental. Desafortunadamente, este proceso arduo que realizaban los alumnos para guardar información, se tornaba difícil, una vez que ellos necesitaban recuperar y utilizar los datos para aplicarlos a la resolución de problemas ligados a su contexto.

En estos modelos de aprendizaje mecanicista, basados en la repetición y memorización de información, el educando ejercía un rol pasivo, era un simple receptor de conocimiento. Se le preparaba para responder a un test, o a lo que Paulo Freire (1980), denominaba como educación bancaria. Por ello, se hizo prioritario buscar otras alternativas de enseñanza-aprendizaje que adjudicase al educando como agente activo.

En la actualidad se han realizado varios estudios sobre la importancia del aprendizaje significativo. Entre ellos, De Zubiría (2006), plantea que es necesario tener otra concepción sobre el aprendizaje, muy diferente a la formación transmisionista, es decir, una educación que garantice que el estudiante sea protagonista activo, capaz de reconstruir conceptos e incorporarlos a sus estructuras de pensamiento para la resolución de problemas que se presenten en su entorno.

Sin duda alguna, la educación ha evolucionado y en la actualidad se espera que el alumno ya no retenga o guarde información de forma exacta, sino que este sea un aprendiz consciente, responsable, constructor de su propio conocimiento. De igual forma, se aspira a que el docente, no solo sea un simple transmisor de información, sino un facilitador que garantice que el estudiante pueda generar nuevos conocimientos.

En este sentido, la teoría clásica del aprendizaje significativo presenta un enfoque que apunta a una enseñanza-aprendizaje con acciones significativas, donde el estudiante atribuye un valor a lo que aprende y un sentido personal al conocimiento. Esta teoría, del siglo XX, propuesta por el psicólogo y pedagogo David Ausubel en los años sesenta $(1963,1968)$ y reiterada en el año 2000, por Moreira, (2017) continúa vigente, por diversos autores. Arias, (2001), por citar alguno, es partidario de la teoría del aprendizaje por

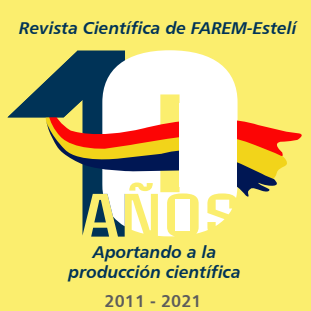


descubrimiento de Bruner (1968), Este pedagogo da a conocer su teoría, según la cual el aprendizaje significativo se contrapone al aprendizaje memorístico, lo cual supone promover la comprensión en vez de la memorización, donde se espera que el alumno adquiera el conocimiento por sí mismo.

Por lo tanto, se tiene como objetivo, en este artículo, recoger los principales planteamientos sobre el aprendizaje significativo aplicado a la educación y analizar el valor que éste tiene en el contexto actual, enfocado de modo particular en la educación superior. Por ello, se realizó un recorrido histórico sobre sus principales postulados, con el propósito de comprobar cómo, a partir de los planteamientos de propuestas pedagógicas como esta, se puede contribuir a una mejor educación.

\section{MATERIALES Y MÉTODOS}

Siendo el presente artículo un texto de revisión bibliográfica, se llevó a cabo una serie de actividades destinadas a localizar y recuperar documentos relacionados con el tema del articulo: "el aprendizaje significativo". Una vez recopilados, los documentos fueron organizados en base a su enfoque y naturaleza. Es decir, se organizaron en base a su contenido y tipo de documento - artículos, ensayos o libros. De igual manera, dichos documentos fueron analizados en base a su confiabilidad y validez para llevar a cabo, subsecuentemente, la discusión y desarrollo de la información a lo largo del presente trabajo.

\section{RESULTADOS Y DISCUSIÓN}

\section{Definición de aprendizaje significativo}

Existen diferentes conceptos sobre aprendizaje significativo. Sin embargo, para el propósito de este artículo de revisión literaria, se define aprendizaje significativo como la relación que existe entre los conocimientos y experiencias previas del estudiante y los nuevos conocimientos (Ausubel, 1980).

Esta conceptualización es pertinente y aplicable al ámbito educativo nicaragüense, en el cual se espera que el alumno pueda utilizar sus conocimientos existentes, fruto de sus experiencias sociales y educativas, al transitar de la educación media a la superior. La mayoría de los estudiantes que ingresan a la educación superior nicaragüense, provienen de escuelas públicas donde pasan cinco años de estudio, muchas veces en un mismo

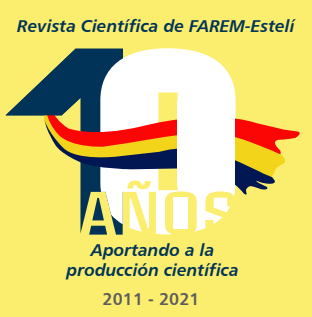


establecimiento. Cada uno de ellos trae consigo un cúmulo de experiencias previas, que se convierten en el punto de partida al iniciar su nuevo aprendizaje en educación superior.

De acuerdo con David Ausubel (1980), la estructura cognitiva que posee el estudiante es trascendental al momento de abordar un nuevo aprendizaje, y el sentido que este le atribuye al objeto de estudio está estrechamente relacionado a las experiencias previas. Los resultados de aprendizaje donde afloran nuevos conocimientos son el reflejo de que se ha consumado un proceso de aprendizaje valioso por parte del estudiante (Ausubel, Novack \& Hanesian, 1983). Por citar un ejemplo, el alumno al transitar de la educación media a la superior enfrenta sentimientos de contradicción y esperanza, desarticulación y discontinuidad entre ambos currículos educativos, dado que el estudiante culmina una etapa e inicia otra a su vez.

En función a lo señalado anteriormente, Ausubel (1980), afirma que es preciso elaborar un material de aprendizaje que no responda al capricho de un programa de estudio, sino que sea elaborado acorde con la estructura cognitiva o proceso mental que permite la organización de dicha información, de tal manera que la persona pueda aprender y recordar. Es decir, estas estructuras integran lo que es la comprensión y la memoria, determinado los conocimientos y conceptos que el alumno posee y su forma de organizarlos en la mente.

Es importante reconocer que al llegar a la educación superior el estudiante no inicia su aprendizaje de cero, sino que hace uso de sus vivencias previas para construir su nuevo conocimiento. A este respecto, los conocimientos previos condicionan, de una u otra forma, lo que los estudiantes aprenden. Estas ideas previas pueden ser aprovechadas por el docente para enriquecer y mejorar el proceso de aprendizaje.

Para resumir lo anteriormente dicho, cuando el alumno llega al salón de clase, posee un mundo de experiencias como producto de sus vivencias en el entorno. El docente, por consiguiente, tiene el trabajo de ayudarle a descubrir y utilizar esos aprendizajes que, muchas veces, ellos desconocen que disponen. Entonces, el docente debe preparar su lección, de manera que esta provea al aprendiz con oportunidades de conciliar el nuevo contenido con lo que él conoce.

\section{Características del Aprendizaje Significativo}

En este modelo, la esencia radica en que el aprendizaje significativo es el resultado de la relación que se establece entre la nueva información y la estructura cognitiva del estudiante, en otras palabras, con lo que el alumno ya sabe (Ausubel, 2000). Este es un proceso en el que se presupone que el alumno tiene una actitud y una disposición para aprender y relacionar 
el material de aprendizaje de que dispone, con su estructura cognitiva, de modo intencional y no al pie de la letra.

En este contexto, UNAN-Managua (2011), (Universidad Nacional Autónoma de Nicaragua) en su fundamentación epistemológica reconoce que: "nuestros conocimientos están influenciados por las experiencias que vivimos, las pasadas y las del contexto actual. El conocimiento es un resultado de las experiencias previas y las situaciones presentes. Donde las experiencias pasadas inciden en la manera que interpretamos el presente" (p.26). Dicho de otra manera, los estudiantes hacen uso de todas aquellas ideas o conceptos que han formado a lo largo de su vida, las que condicionan su forma de interactuar con el nuevo contenido y atribuir valor al mismo.

Para que se logre un aprendizaje significativo, Ausubel (1980) indica que "el material lógicamente debe ser relacionable, no arbitraria, pero sí sustancialmente con ideas pertinentes y correspondientes que se hallen dentro de la capacidad del aprendizaje humano. Esto significa, sencillamente, que si el material en sí muestra la suficiente intencionalidad (o falta de arbitrariedad), entonces hay una manera adecuada y casi obvia de relacionarlo de modo no arbitrario con las clases de ideas y correspondientemente pertinentes que los seres humanos son capaces de aprender" ( p. 4). El conocimiento no se transmite, sino que se construye en la práctica, y el alumno y material de aprendizaje juegan un rol fundamental para que se logre la construcción de nuevos saberes.

En este aspecto, el material de aprendizaje lógicamente significativo requiere un adecuado uso del lenguaje, al establecer relaciones, ejemplos, o ideas pertinentes que sean congruentes con el tema que se imparte. Ausubel (1980) enfatiza en la relacionalidad sustancial, que significa que el material de aprendizaje no debe ser arbitrario, y que es posible utilizarlo de modo tal que pueda interpretarse bajo distintos conceptos o sinónimos, lo que permite al alumno adquirir y comprender nuevos significados. De lo contrario, se continuaría con el modelo de aprendizaje por repetición, estableciendo relaciones de forma arbitraria, aprendizaje que es memorizado por cortos períodos, para después ser olvidado, lo cual trae graves consecuencias para el proceso de enseñanza-aprendizaje.

UNAN-Managua (2011), señala que "el proceso de enseñanza aprendizaje debe buscar interacciones más ricas y significativas entre los diferentes elementos del currículo: estudiantes, docentes, objetivos, contenidos, materiales y recursos" (p.26). En el caso del aprendizaje significativo, el material de aprendizaje tiene un papel preponderante, ya que este provee la oportunidad para que el alumno establezca relación entre el nuevo contenido y sus ideas existentes.

Según Montilla \& Arrieta (2015), existen ciertos requerimientos específicos que deben ser tomados en cuenta para la elaboración de materiales

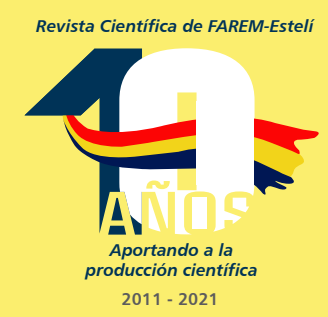


potencialmente significativos. Estos se clasifican en necesarios, que son los que brindan elementos para una potencialidad significativa mínima, y los complementarios, que ofrecen distintos grados de potencialidad al material educativo. En otras palabras, lo que hace que un contenido sea más o menos significativo es su grado de inserción en otros esquemas de conocimiento que el alumno tiene, por ello, es fundamental que la información se presente de manera organizada y articulada con la presencia de diversos tipos de estrategias de aprendizajes.

UNAN-Managua (2011), en su fundamentación pedagógica reconoce que el papel fundamental de la educación superior es "promover la construcción de saberes que tengan significado y relevancia en la solución de problemas reales y cotidianos. En la formación científica y humanística de los estudiantes se propicia la interacción y la autorreflexión, prestando así atención al desarrollo del pensamiento analítico y crítico" (p.27). En tal sentido, es vital mencionar algunas estrategias de enseñanza que favorecen el aprendizaje significativo: los esquemas, los mapas conceptuales, los resúmenes, las preguntas, las analogías, la realimentación, los glosarios, las redes semánticas, etc. (Fernández, 2006). Estas técnicas permiten al estudiante gestionar y organizar mejor su conocimiento para su activación y utilización en la solución de problemas.

Ausubel (2000), establece la diferencia entre dos clases básicas de aprendizaje significativo: El aprendizaje por representaciones, que se ocupa de los significados de símbolos unitarios de palabras, y el aprendizaje de proposiciones, que se refiere a las ideas expresadas por grupos de palabras combinadas en proposiciones u oraciones (p.6). "El tipo básico de aprendizaje significativo, del cual dependen todos los demás aprendizajes de esta clase, es el aprendizaje de representaciones, que consiste en hacerse del significado de símbolos solos (generalmente palabras) o de lo que éstos representan" (Ausubel, 2000, p. 5).

El autor también señala la existencia de una tercera clase de aprendizaje significativo que se basa en la representación de conceptos, entendidos como ideas generales, y organizacionales en categorías. Este, obviamente, se constituye en un nivel superior de aprendizaje de representaciones, puesto que "aprender lo que significa el concepto mismo, que en efecto consiste en aprender cuáles son sus atributos de criterio (los que sirven para distinguirlo o identificarlo), implica un tipo muy diferente de aprendizaje significativo que, como el de proposiciones, es de naturaleza e intención sustantiva en lugar de nominalista o representativa" (Ausubel, 1980, p. 7).

Sin embargo, es oportuno señalar que, en las distintas clases de aprendizaje significativo, se requiere la disposición por parte del estudiante para relacionar el material con el conocimiento que ya posee (Ausubel, 1980). Esta condición significa que, indistintamente de la clase de material empleado para enseñar, si la intención del alumno es simplemente memorizarlo

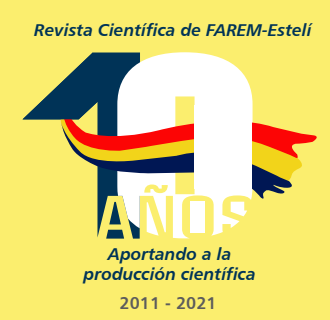


textualmente, el proceso de enseñanza-aprendizaje continuará siendo algo mecánico e intrascendente. Si bien la teoría del aprendizaje significativo les otorga especial atención a los saberes previos del alumno, no desconoce el rol que cumple el docente en la organización del material de enseñanza, de modo pertinente, interrelacionado y substancial.

La teoría del aprendizaje significativo presenta formas de evaluar su eficiencia, como la resolución de problemas que requieran la máxima transformación del conocimiento adquirido y transferencia a nuevas situaciones. Para Moreira (2017), la resolución de problemas es sin duda, un método válido y práctico para buscar evidencias de aprendizaje significativo y según Ausubel (1980), tal vez sea la única manera de evaluar, en ciertas situaciones, si los alumnos realmente comprendieron de manera significativa las ideas que son capaces de verbalizar.

Ausubel (2000) propone "principios programáticos facilitadores como la diferenciación progresiva, la reconciliación integradora, la organización secuencial y la consolidación, y algunas estrategias facilitadoras como los organizadores previos, los mapas conceptuales y los diagramas V" ( citado en Montilla \& Arrieta, 2015, p. 69).

La diferenciación progresiva consiste en presentar las ideas más generales e inclusivas en la etapa inicial de aprendizaje, presentando lo más relevante, para luego de forma progresiva, diferenciarlas mediante ejemplos, situaciones, ejercicios, etc. La reconciliación integradora, por su parte, consiste en "explorar relaciones entre las diferencias y similitudes relevantes, reconciliando inconsistencias reales y aparentes" (Montilla \& Arrieta, 2015, p. 69). Con respecto a la organización secuencial, esta se enfoca en organizar las unidades de estudio de forma coherente, de forma secuencial y la consolidación, es la comprobación de la adquisición de los conocimientos previos antes de abordar otros temas.

Se han presentado algunas características principales del aprendizaje significativo con el fin de que esta teoría pueda ser aplicada en el salón de clase y garantizar así, de esa manera, la adquisición, retención y asimilación de los contenidos que los estudiantes reciben en su escuela, de modo que, estos atribuyan un significado a su aprendizaje.

Adicionalmente, el material también juega un rol vital y debe contar con los subsumidores específicos, es decir, ideas, conceptos o proposiciones inclusivas, claras y adecuadamente estructuradas en la mente del aprendiz, para la incorporación, comprensión y fijación de los nuevos conocimientos (Moreira, 1997). Estas ideas de anclaje facilitaran el aprendizaje subsecuente, proporcionando un nuevo significado para el individuo, con el cual el nuevo material es relacionable (significado psicológico) (Ausubel, 2000).

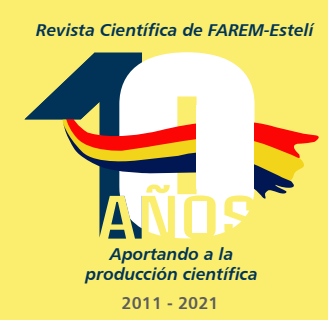


En otras palabras, el material de aprendizaje necesita estar vinculado con la estructura cognitiva del estudiante para ser potencialmente significativo. Así mismo, el alumno debe demostrar actitud y disposición hacia el aprendizaje, de lo contrario este se volverá repetitivo y carente de valor.

Por su parte Moreira, (2000) y Rodríguez, (2008), sostienen que su principal atención radica en lo que acontece dentro del aula cuando el estudiante aprende; la naturaleza del aprendizaje, las condiciones requeridas para que se construya, los resultados de la reestructuración y reorganización de sus ideas respecto a una determinada área y subsiguiente, en la evaluación.

\section{El aprendizaje significativo en la Educación Superior}

En lo que respecta a la educación universitaria, el aprendizaje significativo ofrece herramientas pedagógicas que pueden contribuir a mejorar substancialmente la calidad educativa, bajo la premisa de que "en la utilización de los conocimientos previos radica el sentido y significado que los estudiantes dan a la nueva información para una mayor retención y profundización en la mente de los educandos para que los puedan utilizar en la vida cotidiana" (Asadovay \& Morocho, 2015, p. 2).

El tránsito por los niveles previos a la formación superior (escuela secundaria), constituye una preparación inicial en las distintas áreas del saber, que se aplicarán de modo específico en los estudios profesionales, y, por lo tanto, cada estudiante tiene un acumulado de conocimiento, que le permite elaborar conceptos abstractos, comprender enunciados lógicos, y contar con la capacidad de realizar inducciones y deducciones; es decir, de reflexión sobre distintas clases de información. Bajo esta consideración, es claro que los estudiantes que ingresan a niveles de educación superior, provienen de una etapa diferente.

Por otro lado, existe consenso entre los autores de la literatura consultada, de que hay carencias en la investigación del aprendizaje significativo en distintos niveles educativos, debido, por una parte, a que el modelo adolece de estrategias definidas y competentes para el aprendizaje, y, por otra parte, a que los Centros Universitarios carecen de iniciativas orientadas a un cambio metodológico (Riesgo \& Díaz, 2013).

Asimismo, se están aplicando de manera inadecuada los principios del aprendizaje significativo en los procesos de enseñanza-aprendizaje, tanto a nivel escolarizado como universitario (Baro, 2011). No es remoto, por tanto, encontrarse que, en algunos centros de educación superior, se continúe enseñando bajo modelos tradiciones impositivas y desconectados de la realidad (interna y externa) del educando, con un enfoque memorístico y repetitivo, en lugar de optar por otros métodos pedagógicos como el aprendizaje significativo.

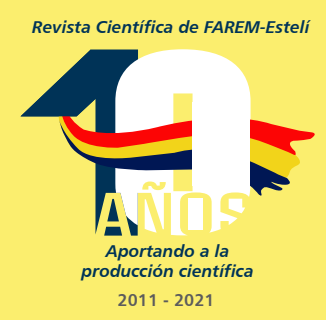


Siendo que la universidad es el centro de formación en la etapa final de la educación y previa a la vida profesional y laboral, es de vital importancia preguntarse por la naturaleza y el sentido de la educación actual, y el impacto que tiene la formación superior en la sociedad. El sentido y la razón de ser de la universidad es, entonces, la de formar seres humanos consecuentes con los problemas de la sociedad, y para ello, demanda estrategias pedagógicas que contribuyan a la formación del espíritu crítico, reflexivo, auto-consciente en los alumnos, en términos de Freire (1980), a una educación emancipadora.

De Soussa Santos (2007), afirma que "la universidad es una de las instituciones fundamentales en la construcción de esa fabulosa ingeniería social que denominamos modernidad. En efecto, si bien ella precede al despunte y desarrollo de la modernidad, es en este período cuando alcanza la hegemonía y la legitimidad que están actualmente en crisis" (p.11).

Esta última es una posición que concuerda con el sentido humanista en el que hace énfasis el aprendizaje significativo, integrando pensamientos, sentimientos y acciones en los educandos, que deben estar presentes en el acto educativo de forma permanente, con el fin de preparar individuos no solo en la adquisición de nuevos conocimientos, en el mundo globalizado e interconectado de hoy en día, sino de conocimientos con significado, comprensión, criticidad y con la posibilidad de usar ese conocimiento en la solución de situaciones problema de modo competente (Novak, 1981).

En la actualidad, son muchas las propuestas de esta naturaleza que están siendo tomadas en cuenta en las universidades, en donde se comienza a tener un enfoque interdisciplinar, más humano, con sentido crítico y reflexivo, consecuente con la realidad propia de los países latinoamericanos, y considerando como referentes los pensadores y teóricos de la educación de nuestro continente.

Si bien, el modelo pedagógico del aprendizaje significativo está orientado a dotar de mayor eficacia el proceso de enseñanza-aprendizaje, no se debe olvidar cuáles son los propósitos últimos de la educación. El fin que debe tener la educación, como práctica emancipadora, resulta ser el más acorde a nuestra condición pasada y presente.

Freire (1980) considera que las prácticas educativas siempre son políticas porque involucran valores, proyectos, utopías que reproducen, legitiman, cuestionan o transforman las relaciones de poder prevalecientes en la sociedad. La educación nunca es neutral, está a favor de la dominación o de la emancipación.

Para Freire, la educación debe servir para que los educadores y los educandos aprendan de su historia y puedan comprender su realidad, pero ello, supone entender críticamente su mundo y actuar para transformarlo en función de su entorno, a través de la acción y reflexión, los educandos y educadores se

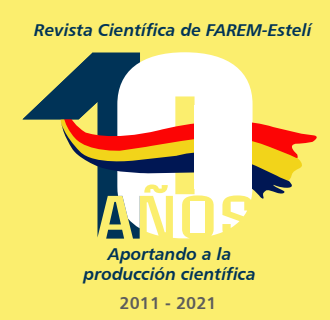


constituyen en sujetos que aprenden mutuamente y le confieren un sentido más significativo al proceso educativo. Esto es algo que guarda concordancia con los planteamientos del aprendizaje significativo, en el que es tan importante el rol del educador, como los conocimientos previos del educando.

Como fruto del proceso natural del pensamiento y de mis observaciones en distintos ámbitos educativos, cabe una reflexión personal, con el fin de aclarar y alcanzar conclusiones sobre el valor del aprendizaje significativo en la educación superior en el contexto nicaragüense, particularmente situado en UNAN-Managua. Al analizar algunos de los factores que todavía tienen presencia y afectan la educación superior, se puede mencionar que, aún persisten en la práctica docente muchas de las características de la pedagogía tradicional, donde el docente se concibe como el principal transmisor de conocimiento y la enseñanza está centrada en él. Se puede decir que, aun se percibe resistencia a los cambios actuales de la educación.

En este modelo tradicional, el estudiante es un receptor pasivo que memoriza y retiene un gran volumen de información para ser utilizada a corto plazo. Aún es común observar que el alumno demuestra poca iniciativa para ser partícipe de las actividades que ocurren dentro del salón de clase y usualmente solo participa cuando el docente se lo solicita.

Por tal razón, es vital, dada las exigencias sociales, culturales y económicas que el mundo demanda, pensar en una educación superior que provea al estudiante con las herramientas necesarias para que este pueda reconstruir los esquemas de conocimiento partiendo de sus vivencias previas. Pero para que se logre este cambio, es trascendental que el docente vea en el estudiante a un ser humano con un cúmulo de experiencias y conocimientos previos producto de su relación con el medio, y parta de esos conceptos para ayudar al alumno en la construcción de nuevos saberes. Sobre todo, la educación superior debe facilitar un espacio para la adquisición de la autonomía intelectual. Para este fin, el docente debe evitar percibir al estudiante como una hoja en blanco, en la cual, se deposita información.

Finalmente, no es desconocido los constantes cambios en la sociedad actual de tipo cultural, social y económico que demandan que la educación superior estructure, modernice sus métodos y programas de estudio. Como solución a esta situación, es una buena alternativa la aplicación de la teoría del aprendizaje significativo, que, a diferencia de la pedagogía tradicional, no ignora los conceptos o ideas que posee el estudiante, sino que parte de las mismas para la construcción de nuevas habilidades, conocimientos y destrezas.

En esta teoría, el estudiante comprende la información que se le provee, existe relación entre la nueva información y la que posee el aprendiz en su estructura cognitiva, el aprendiz decide aprender, siempre y cuando demuestre disposición, y el material de aprendizaje esté organizado de modo

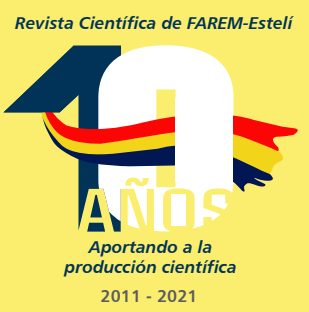


que encuentre significado en el mismo. El docente, por otro lado, elabora el material pertinente a la historia de vida de los estudiantes para que se dé la activación del conocimiento previo y los que aprenden puedan dar al mismo un valor significativo. El maestro se vuelve un auxiliar del estudiante, es decir, un garante que emplea los recursos necesarios para que se facilite la transición del nuevo contenido a la estructura cognitiva del estudiante de manera significativa.

\section{CONCLUSIONES}

En conclusión, la teoría del aprendizaje significativo contiene valores que pueden ser aplicables a la educación superior. En este tipo de aprendizaje el conocimiento previo que trae el alumno al salón de clase es vital para que se logre dar un aprendizaje significativo. De igual forma, el material de aprendizaje tiene un papel preponderante, ya que da las pautas para que el estudiante establezca relación entre lo que él conoce y el nuevo contenido.

Asimismo, los aportes de la teoría del aprendizaje significativo, pueden contribuir mucho más a generar modelos pedagógicos que renueven y transformen los esquemas tradicionales que permitan alcanzar mayores dimensiones y repercusiones en lo social, político, económico, cultural, entre otros aspectos.

Una educación de calidad, humanista, capaz de formar personas conscientes, responsables con iniciativas y que puedan pensar por sí mismas, requiere del reconocimiento de las distintas esferas que integran el conocimiento durante la formación de la persona. Sin embargo, el aprendizaje significativo adolece de estrategias definidas y competentes para el aprendizaje, por lo que se precisa colocar esta propuesta sobre la mesa de discusión y debate, para ser implementada metodológicamente.

\section{REFERENCIAS}

Arias, W. (2001). Sócrates el primer constructivista. Paradigma educativo, $3(5), 11-13$.

Asadovay, D., \& Morocho, L. (2015). La activación de los conocimientos previos para lograr un aprendizaje en niños de E.G.B. Cuenca, Ecuador: ( Tesis de Grado) Universidad de Cuenca, Ecuador.

Ausubel. (1980). Psicología Educativa. Un punto de vista cognoscitivo.

México: 4a. Ed. Trillas disponible en: http://www.educainformatica.com.

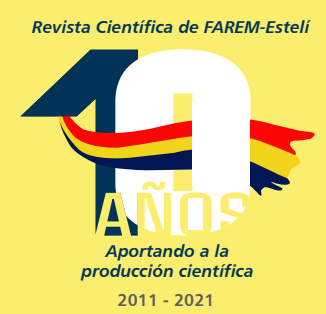


ar/docentes/tuarticulo/educacion/

Ausubel, D. (2000). Adquisición y retención del conocimiento. Una perspectiva cognitiva. Barcelona: Paidós.

Ausubel, Novack \& Hanesian. (1983). Psicología Educativa. Un punto de vista cognoscitivo. México: Ed. Trillas/ disponible en: http://www. educainformatica.com.ar/docentes/tuarticulo/educacion/

Baro, A. (2011). Metodologías activas y aprendizaje por descubrimiento. Revista Digital Innovación y Experiencias Educativas, 40, 1-11.

Bruner. (1968). El proceso de la educación. México: Unión tipográfica Editorial Hispano Americana.

De Zubiría, J. (2006). Los modelos pedagógicos. Hacia una pedagogía dialogante ( $2^{\mathrm{a}}$ ed.). Bogota: Magisterio.

De Soussa Santos, B. (2007). La Universidad en el Siglo XXI / Para una reforma democrática y emancipatoria de la Universidad. La Paz, Bolivia: CIDESUMSA, ASDI y Plural editores, 4 a. ed.

Fernández, N. (2006). Estrategias de enseñanza para favorecer el aprendizaje significativo. cognición, 5, 12-18.

Freire, P. (1980). Pedagogía del Oprimido. Bogotá, Colombia: Siglo XXI editores, 24a. ed.

Montilla, R., \& Arrieta, X. (2015). Secuencia didáctica para el aprendizaje significativo del análisis volumétrico. Omnia / Universidad del Zulia, Maracaibo, Venezuela, vol. 21, núm. 1, enero-abril, pp. 66-79. Disponible en: http://www.redalyc.org/articulo.oa? id =73742121006

Moreira, M. (1997). Aprendizaje significativo: un concepto subyacente. Burgos: España.

Moreira, M. (2000). Aprendizaje significativo. teoría y práctica. Madrid: Ed. Visor.

Moreira, M. (2017). Aprendizaje significativo como un referente para la organización de la enseñanza. Archivo de las ciencias de la educación, Vo. 11 No. 12, e29 / Disponible en: http://www.memoria.fahce.unlp. edu.ar/art_revistas/pr.8290/pr.8290.pdf.

Novak, J. (1981). Una teoría de la educación. Sao Paulo, Brasil: Pionera.

Riesgo, M., \& Díaz, M. (2013). Reinventando la rueda: una experiencia de aprendizaje por descubrimiento enla asignatura de sistemas operativos. Revisión, 6(1), 12-19.

UNAN-Managua. (2011). Modelo educativo, normativa y metodología para la planificación curricular.

Vigotsky, L. (1978). Pensamiento y Lenguaje. Barcelona, España: Ediciones Fausto. 